\title{
$O$ impacto do desenvolvimento de transtornos alimentares em adolescentes: uma
}

\section{revisão}

\author{
The impact of the development of eating disorders in adolescents: a review \\ El impacto del desarrollo de los transtornos alimentarios em los adolescentes: una revisión
}

Recebido: 11/10/2021 | Revisado: 18/10/2021 | Aceito: 24/10/2021 | Publicado: 25/10/2021

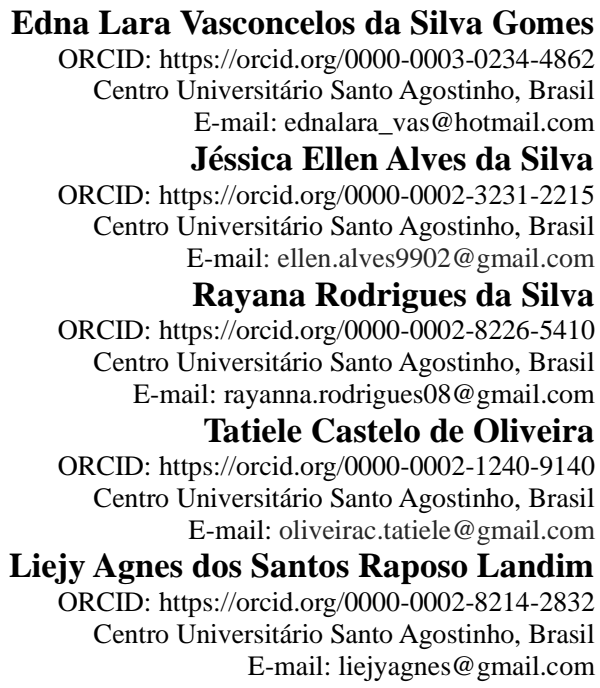

\begin{abstract}
Resumo
Os transtornos alimentares caracterizam-se por quadros psiquiátricos que resultam em complicações clínicas e tem como danos à saúde alterações no comportamento alimentar, severas distorções em sua imagem corporal e agravos psicossociais. O presente estudo objetivou realizar uma revisão narrativa sobre o impacto do desenvolvimento de transtornos alimentares em adolescentes. Os descritores utilizados durante a pesquisa foram: "Transtorno Alimentar", "Adolescentes" e "Hábitos Alimentares". A pesquisa foi realizada nas bases de dados BVS, CAPES, LILACS, PUBMED e SCIELO. Os resultados revelaram que o impacto causado nos adolescentes pelos transtornos alimentares são causas como obesidade, desnutrição e mortalidade. A prevalência tem incidência maior no sexo feminino independente da classe econômica-social. Nesse sentido, salienta-se a importância do diagnóstico precoce, em conjunto a uma equipe multiprofissional, no qual faz-se necessário a presença de um profissional nutricionista especialista na área de transtornos alimentares.
\end{abstract}

Palavras-chave: Transtorno alimentar; Adolescentes; Hábitos alimentares.

\begin{abstract}
The eating disorders are characterized by psychiatric condition that result in clinical complications and have as damages to health changes in eating behavior, severe distortions in body image and psychosocial agravations. The study aimed to perform a narrative review on the impact of the development of eating disorders in adolescents, the descriptors used during the search were "Eating Disorder", "Adolescent", "Eating Habits". The search was carried out in the BVS, CAPES, LILACS, PUBMED and malnutrition and mortality. The prevalence has a higher incidence in females regardless of social-economic class. In this sense we emphasize the importance of early diagnosis, together with a multiprofessional team, in which the presence of a nutritionist professional specialized in the ED area is necessary.
\end{abstract}

Keywords: Eating disorder; Adolescent; Eating habits.

\section{Resumen}

Los transtornos alimentarios se caracterizan por condiciones psiquiatricas que resutan en complicaciones clinicas y tienen como daño a la salud combios en la conducta alimentaria, graves distorsiones en su imagen corporal y problemas psicosociales. El estudio tuvo como objetivo realizar una revisión narrativa sobre el impacto del desarrollo de los transtornos alimentarios en adolescentes. Los descriptores utilizados dursnte la investigación fueron: "Desorden Alimenticio", "Adolescente", "Hábitos Alimenticios". La investigación se realizó en las bases de dados BVS, CAPES, 
LILACS, PUBMED y SCIELO. Los resultados revelaron que el impacto que provocan en los adolescentes los transtornos alimentarios son causas como la obesidad la desnutrición y la mortalidad. La prevalencia tiene una mayor incidencia en mujeres independientemente de la clase economica y social. En este sentido, la importancia del diagnostico precoz junto con un equipo multidisciplinar, en el que es necesaria la presencia de un nutricionista profesinal especializado en el campo de la T.A.

Palabras clave: Desorden alimenticio; Adolescente; Hábitos alimenticios.

\section{Introdução}

A adolescência é um período marcado por diversas mudanças, tanto fisiológicas quanto bioquímicas, no qual os adolescentes são marcados pela puberdade e ganho de peso, principalmente as meninas, e todas as alterações hormonais e corporais tornam-se ainda mais evidentes, caracterizando, dessa forma, um período delicado para um possível caso de Transtorno Alimentar (TA) (Lima, Rosa, \& Rosa, 2012).

Os transtornos alimentares (TAs) consistem em quadros psiquiátricos resultante de complicações clínicas e tem como danos á saúde alteraç̃̃es no comportamento alimentar, severas distorç̃̃es em sua imagem corporal e agravos psicossociais, os quais levam a uma série de atitudes comportamentais compensatórias como a inanição e/ou compulsão purgação restrição, ocasionando, desse modo, os diagnósticos de TAs, como anorexia nervosa (AN), bulimia nervosa (BN) e compulsão alimentar (CA), sendo esses os principais e que mais acometem os jovens (Alvarenga, 2017).

A origem dos TAs é multifatorial, envolvem aspectos biológicos (obesidade e sexo feminino), personalidade e socioculturais (culturas, ambiente em meio no qual o adolescente está inserido). Com isso, a conduta de pacientes acometidos por algum TA é delicada, bem como não apresenta um bom prognóstico, devido muitas vezes à falta de entrega do paciente ao tratamento, assim levando a altas taxas de recorrências e a elevados índices de mortalidade devido a casos de suicídio, e casos de broncoaspiração, desequilíbrios hídricos e àcido-base causados em decorrência da bulimia nervosa. (Gonzalez et al., 2019).

No que se refere a compulsão alimentar ou transtorno de compulsão alimentar (TCA) a American Psychiatric Association (2014, p. 350) descreve com critérios para diagnostico, episódios recorrentes de compulsão alimentar com ingestão de alimentos em um período limitado (exemplo, a cada 2 horas) com uma quantidade de alimentos muito grandes que a capacidade fisiológica normalmente aceitaria no mesmo período de tempo, com isso um sentimento de falta de controle sobre a situação, o individuo não consegue parar de comer ou controlar o seu ato, sendo a ação executada com grandes quantidades de alimento, onde o sujeito come até sentir-se extremamente incomodado, além de comer sozinho devido a vergonha que senti ao ingerir uma quantidade exacerbada de comida, após o episódio sente uma sensação de angustia. Ao contrário da anorexia nervosa e bulimia nervosa, o TCA não tem atitudes de purgação.

Em contrapartida ao cenário dos TAs, como medidas preventivas em alguns casos e até mesmo medidas de tratamento, utilizam-se estratégias que contribuem com a melhora dos pacientes, sendo utilizados condutadas de multiprofissionais onde incluem-se nesse quadro nutricionistas, psiquiatras, nutrólogos, psicólogos, educador físico, fisioterapeuta, afim de otimizar o tratamento e contribuir com a mudança na percepção de imagem pessoal e corporal que cada paciente tem de si mesmo (Moreira, Boff, Pessa, \& Neufield, 2017).

Diante do contexto explanado, o principal objetivo desse estudo foi realizar uma revisão narrativa sobre o impacto de desenvolvimento de transtornos alimentares em adolescentes, e assim fomentar com pesquisas sobre transtornos alimentares.

\section{Metodologia}

O presente estudo trata-se de uma pesquisa exploratória, desenvolvida através de uma narrativa acerca dos transtornos alimentares mais recorrentes em adolescentes. Fundamentou-se uma análise de artigos científicos obtidos através das bases de dados: Biblioteca Virtual em Saúde (BVS), Coordenação de Aperfeiçoamento de Pessoal de Nível Superior (CAPES), 
Literatura Latino-americana e do Caribe em Ciências da Saúde (LILACS), National Library of Medicine (PUBMED) e Scientific Electronic Library Online (SCIELO), em setembro de 2021, quanto aos indexadores, foram utilizados os termos "Transtornos Alimentares", “Adolescente", "Hábitos Alimentares", e seus respectivos correspondentes em inglês "Eating Disorders", "Adolescent", "Eating Habits", e em espanhol "Desorden Alimenticio", “Adolescente", "Hábitos Alimenticios". Nessa busca foram incluídos tanto artigos em inglês e espanhol quanto em português.

Após a pesquisa nas bases de dados, foram então encontrados o total de 28 artigos, onde logo em seguida procedeu-se a aplicação dos critérios de inclusão, que correspondem ao recorte temporal em relação ao tema pesquisado. Os critérios para exclusão foram, artigos muito antigos sem relação com o tema, e com recorte temporal maior que 07 anos. Neste processo foram então excluídos um total de 04 artigos e incluídos 24 artigos. Desses que cumpriram os critérios de inclusão 20 eram do idioma português, 02 ingleses e 02 em espanhol.

Ambos com publicações com recorte temporal de 2015 a 2021, após o estudo e leitura dos artigos encontrados, a linha de pesquisa em comum acordo foram de 17 artigos, sendo esses utilizados para a discussão deste estudo.

\section{Resultados e Discussão}

\section{Prevalências dos transtornos alimentares na adolescência}

De acordo com estudos realizados notou-se que em relação aos sintomas comportamentais, condutas compensatórias de controle de peso geralmente têm início a partir da anorexia nervosa, bulimia nervosa, transtorno purgativo e transtorno de compulsão alimentar periódica, com a supervalorização do peso, simultaneamente com a sensação de gordura e medo de ganho de peso. A preocupação com o corpo, com as medidas e estar dentro de um "padrão" ditado pela mídia é o ponto ápice para gerar os transtornos citados (Stice et al., 2021).

Dessa forma, observa-se que os adolescentes tendem a ser o grupo com maior risco para o desenvolvimento de um transtorno alimentar. Os sinais característicos dos TAs têm uma alta prevalência na adolescência, sendo considerados precursores importantes de achados clínicos. Evidencia-se esse fato em um estudo longitudinal com 1.528 alunos do ensino médio, onde eles preencheram um questionário de autoavaliação (50,5\% mulheres, onde a maioria tinha idade $=15$ anos). Observou-se que as classes que apresentaram mais sintomas de TAs, sofreram com problemas de identidade, menos esforço no controle e sintomas de internalização. Com isso o estudo ressalta a importância de um diagnóstico precoce em jovens que tendenciam a terem TAs, jovens vulneráveis que apresentam sintomas de TAs (Verscheren et al., 2020).

O sexo feminino é o público mais atingido, os antecedentes familiares também contam como uma elevada probabilidade para o desenvolvimento de doenças crônicas. Quando associamos o conjunto de alimentação inadequada e irregular, com sedentarismo temos como resultado jovens com obesidade, sobrepeso e em contrapartida desnutridos, se tornando um gatilho para o TA. Tendo em vista a cobrança por estar alinhada, adequada, bela, e dentro do padrão estabelecido nas comunidades e até mesmo no ciclo familiar, observa-se essa cobrança em uma demanda maior para as meninas, que por muitas vezes recebem mais exigências do que orientações sobre seu corpo, seus hábitos e comportamentos (Alejandro et al., 2018).

A parcela que se destaca de indivíduos diagnosticados com algum tipo de TA, na maioria dos casos são adolescentes do sexo feminino, e por muitas vezes de alto nível socioeconômico-cultural. Ainda assim, também existem evidências de casos de TAs em adolescentes do sexo masculino, onde os que mais são acometidos são meninos de nível socioeconômico-cultural baixo, raça negra, com idades entre 12 a 25 anos (Ludewig, Rech, Halpern, Zanol, \& Frata, 2017). Em termos de proporções, predominam os casos diagnosticados no sexo feminino, enquanto a cada 3 meninas, 1 menino é diagnosticado (Hercowitz, 2015).

O estudo realizado por Salomão et al., (2021) com um grupo de escolares mostrou uma prevalência de TA em 
adolescentes do sexo feminino e de baixa renda, evidenciando desta forma, a relação socioeconômica e a falta de conhecimento quando associados a urbanização, através da divulgação em massa de ideais de beleza realizadas ao corpo. Em contrapartida, Ludewig et al., (2017) relata em seu estudo transversal que o grupo de meninas diagnosticadas com TA, por muitas vezes é de alto nível socioeconômico-cultural. Dessa forma observa-se que independente da classe econômica-social a prevalência de TA ocorre no sexo feminino, como visto em grande parte dos estudos encontrados na literatura.

\section{Fatores que influenciam o desenvolvimento dos Transtornos Alimentares na adolescência.}

A imagem corporal pode ser definida como a figura mental do corpo; em outras palavras, é o modo pelo qual o corpo se apresenta e como o sujeito a vivência. Dentre as principais influências sofridas pelos indivíduos com relação à imagem corporal, de acordo com o estudo de (Amaral et al., 2019). Contribuindo a essa linha de raciocínio Copetti e Quiroga (2018) destacam como influenciadores as mídias, dando ênfase a televisão e internet como principais veículos influenciadores dos TAs pelo segmento digital, devido a ação do marketing e das grandes indústrias de moda e dietistas que divulgam e valorizam o corpo perfeito.

Tendo em vista o mundo contemporâneo e globalizado, observa-se que os jovens gastam uma grande parcela do seu tempo diário nos meios digitas, onde muitas vezes o conteúdo consumido não é supervisionado por seus responsáveis. Leva-se em consideração a falta de diálogo e orientação dos familiares, quanto a essas questões e outras que não fazem parte desse estudo, o ponto em questão é a ótica da família como o primeiro meio de informação e segurança de acesso aos jovens.

Souza e Pessa (2016), relatam que a etiologia da doença é multifatorial, e com isso desencadeia multe questões que envolvem o convívio do jovem com a sociedade, com a família, a exposição as mídias sociais, assim também como a influência de uma possível pré-disposição genética, e a fase de questionamento do jovem devido a evolução de criança para adolescente. Em complemento a essa linha de raciocínio Timerman (2021), aborda essas influências tendo em vista os grupos sociais, os meios de comunicação em massa, e a dinâmica com o convívio familiar. São conjuntos que se pesados, tornam-se os fatores principais das causas de transtornos alimentares nos jovens.

Destaca-se também como fator de causa, o período em que a puberdade se inicia, devido as alterações morfológicas e psicossociais evidenciarem-se, e com isso leva o jovem a preocupar-se com sua imagem corporal (Martins et al., 2015). Tendo essas informações em mente se torna claro como o meio no qual o jovem está associado, influência diretamente de maneira positiva ou negativa em seus hábitos e costumes.

O diagnóstico precoce dos TAs ou a identificação de riscos para o desenvolvimento dos mesmos nessa fase da vida contribui para que esse problema seja solucionado o mais rápido possível evitando evolução para a fase adulta, reduzindo dessa forma os quadros de desnutrição e mortalidade. Pesquisas como a de Serra e Oliveira (2018) têm buscado valorizar o construto da imagem corporal positiva, o que representa grande avanço nos estudos, visto que essa perspectiva considera a imagem corporal para além de seus aspectos patológicos.

\section{Conduta Multidisciplinar frente aos transtornos Alimentares na adolescência}

O tratamento dos transtornos alimentares envolve medidas não farmacológicas, tendo principalmente como medidas de tratamento modificações ambientais, intervenção psicoterápica individual e familiar, e a terapia cognitivo-comportamental (TCC) esta é utilizada como tratamento na fobia alimentar. Existem relatos de que o uso de inibidores seletivos de receptação da serotonina (ISRS), quando utilizada pequenas doses em crianças com fobia alimentar os mesmos apresentaram melhora significativa. (Da costa, Romano, De morais, 2019). Com isso vemos a importância de uma intervenção multiprofissional para o tratamento de casos como TAs, uma conduta pensada em comum acordo e planejada de forma individualizada e estratégica para cada caso em específico, onde contemplam profissionais nutricionistas, psicólogos, médicos psiquiatras e 
endocrinologistas, fisioterapeutas e educadores físicos.

Os transtornos alimentares têm sido estudados tanto nos segmentos da medicina, biologia, sociologia e psicologia, dito isso, o estudo de Farah e Mate., (2015) também classifica o TA como transtorno dimórfico corporal. Estudos revelam que existe ausência de medidas preventivas, tornando-se necessário mais ações e pesquisas que possibilitem a realização de medidas profiláticas afim de tornar claro os fatores de risco que esses diversos tipos de transtornos podem causar na população jovem.

Assim como complementa o estudo de Timerman., (2021) onde é colocado que é necessário uma maior compreensão sobre os casos de transtornos alimentares, tendo em vista a complexidade, gravidade e consequências. Assim como a importância do senso crítico e da abordagem com os casos, no momento do manejo e transmissão de informações, pois em alguns casos pode ocorrer conceitos extremistas em pacientes com complexos do seu corpo e do seu ato de alimentar-se.

Quanto ao papel do profissional de nutrição frente a tratativa dos transtornos, o estudo de Moraes et al., (2019) mostra que a conduta requer atenção e especialização do nutricionista, onde os manuais recomendam que o tratamento seja levado por nutricionistas especialistas na área, tendo em vista a especificidade e delicadeza dos casos quando se trata de transtornos. A conduta do profissional visa uma intervenção pacífica, onde não é abordado regras rígidas como as dietas, e sim uma abordagem como principal meta o aconselhamento nutricional, essa conduta permite o profissional atentar-se as percepções e pensamentos de como o paciente lida com seus hábitos alimentares.

A intervenção nutricional no TA deve ser a longo prazo, aliado à educação alimentar, preconiza-se que o paciente cumpra metas individuais e especificas, com o objetivo de mudanças sustentáveis e graduais, já que no tratamento não se utiliza dietas. Com isso o papel do nutricionista de forma geral visa auxiliar o paciente com sua relação frente aos alimentos e ao ato de alimenta-se e conduz essa tarefa de forma a orientar, monitorar e dar suporte psicossocial e reforço positivo. Sendo assim tendo como intenção principal evitar danos futuros a saúde do jovem e eventualmente o tornar um adulto saudável.

\section{Considerações Finais}

Em síntese, compreende-se a importância do diagnóstico precoce dos distúrbios, tendo em vista os impactos causados pelos transtornos alimentares nos adolescentes, como casos de obesidade, desnutrição e mortalidade, bem como complicações clínicas causadas pelos distúrbios na alimentação citados no presente estudo. Observa-se a prevalência do sexo feminino nos diagnósticos das complicações, independente da classe social. Está claro que os fatores de maior influência nos casos de TAs são os meios (sociedade, amigos e família) nos quais o indivíduo está inserido, utilização em massa de redes sociais e de todos esses o que está diretamente ligado aos jovens é a dinâmica familiar, onde há uma falha no diálogo e orientação da família para com os jovens.

No que se refere à tratamento, para casos de TAs a conduta é multiprofissional, em que é necessário a presença de um profissional nutricionista nessa equipe. A atuação nutricional desempenha um papel importante, desde que o tratamento seja levado por um nutricionista especializado na área de transtornos alimentares, tendo em vista o seu importante papel em casos que são específicos e delicados. A atuação desse especialista requer a responsabilidade de uma avaliação do estado nutricional e intervenções nutricionais necessárias em cada caso, onde o mesmo deve prestar essa atenção de forma leve, sustentável e com reforço positivo, para melhor entender e contribuir com a saúde do paciente. Enfatiza-se a necessidade de mais pesquisas nesse meio que possibilitem a realização de ações para orientação nutricional acerca de fatores de risco que podem desencadear os diversos tipos de TAs na população jovem. 


\section{Referências}

Amaral, A. C. S. et al. (2019). Apreciação corporal e aspectos associados entre adolescentes e mulheres jovens. Jornal Brasileiro de Psiquiatria, 68, 16-22.

Alvarenga, M. S. (2017). Abordagens no transtorno alimentar - anorexia, bulimia, compulsão alimentar não especificado. In: Trecco, S. (2017). Educação Alimentar e Nutricional: da teoria à prática. Roca. 193-199.

APA. (2014). DSM-5: Manual diagnóstico e estatístico de transtornos mentais. American Psychiatric Association (APA). (5a ed.) Artmed.

Braga, P. D., Molina, M. D. C. B., \& Figueiredo, T. A. M.D. (2018). Representações do corpo: com a palavra um grupo de adolescentes de classes populares. Ciência \& Saúde Coletiva. 23(8).

Copetti, S. V. A., \& Quiroga, V. C. (2020) A influência da mídia nos transtornos alimentares e na autoimagem em adolescentes. Revista de Psicologia da IMED, 10(2), 161-177.

Da costa, V. Z. C., Romano, G. M. K., \& De morais, B. C. M. R. (2019). CAPÍTULO 2 Transtornos da Alimentação. Transtornos alimentares na infância e na adolescência: Uma visão multidisciplinar.

De lima, N. L. et al. (2012) Identificação de fatores de predisposição aos transtornos alimentares: anorexia e bulimia em adolescentes de Belo Horizonte, Minas Gerais. Estudos e Pesquisas em Psicologia, 12(2), 360-378.

Farah, M. H. S., \& Mate, C. H. A. (2015). Uma discussão sobre as práticas de anorexia e bulimia como estéticas de existência. Educação e Pesquisa. 41(4), 883-898.

Gonzales, M. et al. (2019). Transtornos alimentares: critérios diagnósticos, quadro clínico e complicações, epidemiologia e etiologia. In: Alvarenga, M; Dunker, K.L.L., Philippi, S.T. (org). Transtornos alimentares e nutrição: da prevenção ao tratamento. Barueri: Manole.

Timerman, F. (2021). Transtornos alimentares. Editora Senac.

Hercowitz, A. (2015). Transtornos alimentares na adolescência. Pediatria Moderna. 51(7).

Ludewig, A. M. et al. (2017). Prevalência de sintomas para transtornos alimentares em escolares de 11 a 15 anos da rede municipal de ensino da cidade de Nova Petrópolis, RS. Revista da AMRIGS. 61(1), 35-39.

Martins, C. R. et al. (2010). Insatisfação com a imagem corporal e relação com estado nutricional, adiposidade corporal e sintomas de anorexia e bulimia em adolescentes. Rev. Psiquiatr. Rio Gd. Sul. 32(1), 19-23.

Moreira, X. S. G. et al. (2017). Terapia Cognitivo-Comportamental para adolescentes: uma perspectiva transdiagnóstica e desenvolvimental. Alimentação e Imagem Corporal. 150-187.

Moraes, C. E. F., Maravalhas, R. A., \& Mourilhe, C. (2019). O papel do nutricionista na avaliação e tratamento dos transtornos alimentares. Debates em Psiquiatria, Rio de Janeiro. 9(3), 24-30. 10.25118/2763-9037.2019.v9.51. https://revistardp.org.br/revista/article/view/51.

Salomão, B. J. et al. (2021). Indícios de transtornos alimentares em adolescentes. Brazilian Journal of Health Review. 4(2), $5665-5678$.

Serra, V. M. et al. (2018). Prevalência de comportamento de risco para compulsão alimentar em adolescentes de um colégio particular em São Luís-MA. RBONE-Revista Brasileira de Obesidade, Nutrição e Emagrecimento. 12(76), 1029-1038.

Souza, A. P. L. D., \& Pessa, R. P. (2016). Tratamento dos transtornos alimentares: fatores associados ao abandono. Jornal Brasileiro de Psiquiatria, 65, 6067.

Stice, E. et al. (2021). Sequenciamento do surgimento de sintomas na anorexia nervosa, bulimia nervosa, transtorno da compulsão alimentar periódica e transtorno purgativo e as relações dos sintomas prodrômicos com o início futuro desses transtornos. Jornal de Psicologia Anormal. 130(4), $377-2021$.

Verschueren, M. et al. (2020). Sintomatologia do transtorno alimentar em meninos e meninas adolescentes: Identificando classes distintas de trajetória de desenvolvimento. Jornal da Juventude e Adolescência. 49(2), 410-426. 